\title{
ReMed - speziell auf Ärztinnen und Ärzte ausgerichtet
}

\author{
Wenn Ärztinnen und Ärzte selbst einmal Hilfe benötigen, gestehen sich das viele \\ nicht ein - oder erst sehr spät. Dabei ist das Unterstützungsnetzwerk ReMed spezi- \\ ell auf ihre Bedürfnisse ausgerichtet. Es begleitet auf dem Weg aus der Krise und \\ zeigt sowohl im Berufsalltag wie auch im Privatleben Handlungsmöglichkeiten auf.
}

Jürg Bänninger

Leitungsausschuss ReMed

Korrespondenz:

Sekretariat ReMed Postfach 55

CH-3000 Bern 15

Tel. 0313591200

info@swiss-remed.ch

\section{Was sich dank ReMed verändert hat}

Eine 50-jährige Ärztin, in einer Gruppenpraxis, alleinerziehend, berichtet über ihre Erfahrungen mit ReMed:

Eine schwierige private Situation, während der meine Tochter auch noch schwer verunfallte, brachte mich aus dem Gleichgewicht. Ich wurde depressiv, konnte nicht mehr schlafen, richtete im Beruf und privat mit den Terminen ein heilloses Durcheinander an. Krankengeschichten waren plötzlich nicht mehr auffindbar, und Berichte blieben liegen. Auch die Finanzen gerieten aus dem Ruder.

Da beschloss ich, mich bei ReMed zu melden. $\mathrm{Zu}$ meinem Erstaunen hörte mir einfach jemand zu und hatte Zeit für mich - das war schon eine Erleichterung. Ich spürte, dass dieser Kollege weiss, wovon ich spreche, und dass ich ihm meine Situation nicht lange zu erklären brauchte. Es half mir sehr, dass wir alle mir eigentlich bekannten Informationen einmal übersichtlich ordneten und ich dann Prioritäten setzen konnte.

Mein Berater zeigte mir klipp und klar, dass die Belastungen zu gross waren: «Das kann man nicht alleine schaffen!» Ich musste mir eingestehen, dass ich an meine Grenzen gestossen war. Es wurde mir auch bewusst, dass ich überhaupt nicht effizient arbeitete. Seither nehme ich ein Antidepressivum und behandle mein bekanntes ADS wieder mit einer minimalen Dosis Ritalin. So habe ich keine grossen Stimmungsschwankungen mehr und kann viel konzentrierter arbeiten.

Grosse Änderungen gibt es vor allem in der Administration: Meine Tochter kümmert sich jetzt um die Rechnungen, so dass ich mich nicht mehr damit beschäftigen muss und sie etwas Geld verdient. Nun sind wir mit der Rechnungsstellung immer à jour, was sich natürlich auch positiv auf die Liquidität auswirkt. In der Praxis haben wir eine Praktikantin (Sozialbegleiterin) angestellt, die uns bei aufwendigen psychosozialen Problemen unterstützt und uns auch administrativ sehr viel abnimmt. Vereinfacht habe ich auch den Jahresabschluss, den jetzt der Treuhän- der erledigt. Und die Daten übertrage ich neuerdings elektronisch.

Meine Zeit ist viel besser investiert mit verrechenbarer Tätigkeit, vor allem in der Sprechstunde. «Sparen durch Gratisarbeit» ist völlig kontraproduktiv die Effizienz leidet darunter, und es führt nur zur Erschöpfung. Am Telefon grenze ich mich heute viel konsequenter ab. Viele Auskünfte gebe ich zeitsparend per E-Mail. Wenn ich denke, wie viel Zeit ich früher dafür aufgewendet habe!

Die Frage des Kollegen: «Wo ist denn da die Partnerschaft?» hat mich berührt, und ich bin kurz darauf mit meinem Freund für eine Woche ans Meer gefahren. Endlich wieder einmal ein paar Tage Privatleben ohne Alltagssorgen. Es gibt aber noch viel nachzuholen, das weiss ich. Ja, ich merke, ich packe seit dem Kontakt mit ReMed vieles anders an: Ich bin auf dem richtigen Weg. Klar ist, dass ich weiterhin sehr achtsam und konsequent sein muss, sonst falle ich unweigerlich wieder in meine alten Muster.

\section{Unterstützung}

Befinden Sie sich in einer schwierigen Situation und suchen Rat? Oder kennen Sie einen Arzt in Ihrem Umfeld, der Unterstützung benötigt? Dann melden Sie sich bei ReMed über die 24-Stunden-Hotline: 0800073633 oder über help@swiss-remed.ch.

\section{Informationen}

Möchten Sie mehr wissen und auch Ihre Kolleginnen und Kollegen auf ReMed aufmerksam machen? Flyer und Kleinplakate auf Deutsch oder Französisch können Sie kostenlos über info@ swiss-remed.ch anfordern. Zusätzliche Informationen finden Sie auch auf www.swiss-remed.ch

\section{Intervision für Ärztinnen und Ärzte, die mit Kolleginnen und Kollegen arbeiten}

Als Unterstützungsnetzwerk begleitet ReMed nicht nur Ärztinnen und Ärzte in Krisen, sondern 
fördert auch die Vernetzung von Ärztinnen und Ärzten, die ratsuchende Kolleginnen und Kollegen begleiten

ReMed ergriff deshalb die Initiative zur Bildung einer kollegialen Intervision. Die ersten Treffen fanden bereits statt. Es zeigte sich, dass Interesse an der Bildung von halboffenen bis geschlossenen Peer-Groups besteht, die gemeinsam Fallfragen zu Mentoring, Coaching, Beratung oder Therapie, respektive Assessment oder anderen Aspekten (juristisch, versicherungsrechtlich usw.) erarbeiten.

In der Einstiegsphase moderiert ReMed die Gruppen. Später kann die Moderation auch durch ein Gruppenmitglied (abwechselnd oder für eine bestimmte Zeitdauer) übernommen werden. Inhalte sind die fallbezogenen Erfahrungen, Fragen und Anliegen der Anwesenden. Teilnahmevoraussetzung an den Treffen ist Beratungs- bzw. Behandlungserfah- www.swiss-remed.ch - ora anche in italiano ReMed ha ampliato la sua offerta informativa: ora su www.swiss-remed.ch sono disponibili tutte le informazioni anche in italiano. Dia un'occhiata.

www.swiss-remed.ch - now also in English ReMed has expanded its range of information services: www.swiss-remed.ch now provides all information in English. Check it out.

für die Teilnehmenden ausser den Reisekosten grundsätzlich keine. Sollte ein Supervisor oder ein anderer Spezialist eingeladen werden, geschieht dies im Auftrag der Gruppe, die dann auch die Kosten übernimmt.

\section{Die nächsten Intervisionstreffen finden am 7. Juli und 3. November statt.}

rung mit ärztlichen Kolleginnen und Kollegen. Die Gruppe kann auch Personen aufnehmen, die andere hier nicht genannte Aspekte einbringen können und/ oder nicht ärztlich tätig sind.

Die Treffen werden ca. 3 bis 4 Mal pro Jahr rotierend bei den einzelnen Teilnehmenden stattfinden und abhängig von der Interessenlage der Teilnehmenden 3 bis 4 Stunden dauern. Kosten entstehen
Die Einhaltung der Schweigepflicht ist für alle Teilnehmenden verbindlich. $\mathrm{Zu}$ beachten ist weiter, dass die Teilnahme an der Intervision keine Fallüberweisung garantiert.

Die nächsten Intervisionstreffen finden an folgenden Daten statt: 7. Juli 2011 und 3. November 2011. Anmeldungen nimmt ReMed gerne entgegen unter: info@swiss-remed.ch

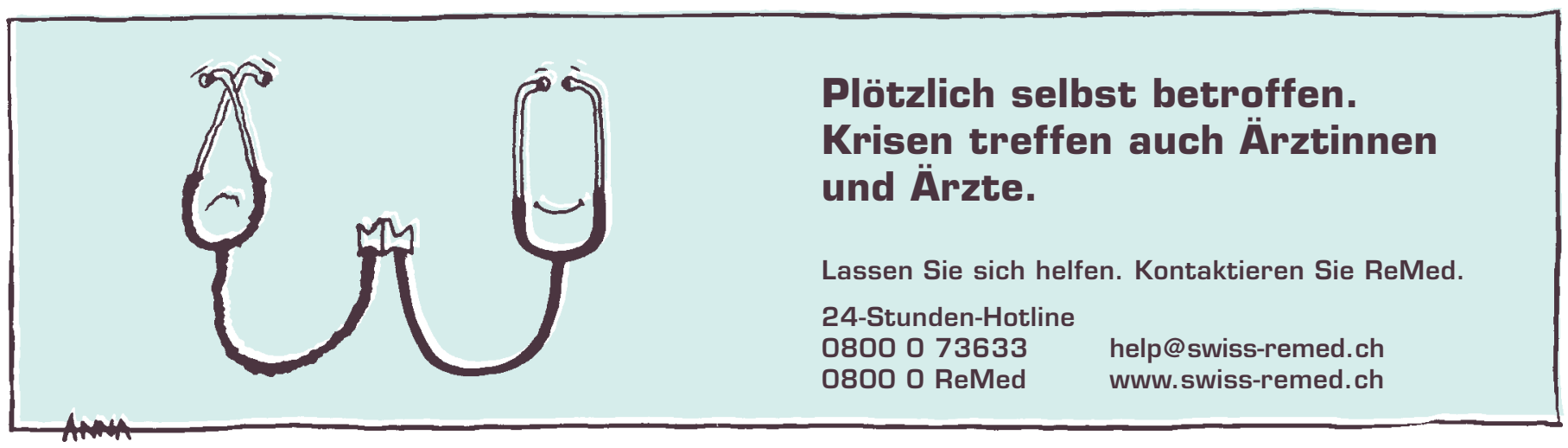

